\title{
EFFECTS OF ALPHA-S1-CASEIN TRYPTIC HYDROLYSATE AND L-THEANINE ON SLEEP DISORDER AND PSYCHOLOGICAL COMPONENTS: A RANDOMIZED, DOUBLE-BLIND, PLACEBO-CONTROLLED STUDY
}

\author{
Chee Huei Phing ${ }^{1}$ and Ong Yong Chee ${ }^{2}$ \\ ${ }^{1}$ Faculty of Science, Universiti Tunku Abdul Rahman, Kampar, Perak, Malaysia \\ ${ }^{2}$ Ministry of Health, Malaysia
}

Corresponding author: Chee Huei Phing

Email: cheehp@utar.edu.my

\begin{abstract}
Sleep disorder is common among the general population. A nutritional supplement containing alpha-s1-casein tryptic hydrolysate and L-theanine was evaluated for its effects in improving sleep quality. The study was conducted using a double-blind randomized trial. Intervention group received once-daily capsules of $150 \mathrm{mg}$ alpha s1 casein tryptic hydrolysate and $50 \mathrm{mg}$ L-theanine, and the control group were given placebo (150 mg skimmed milk powder) for four weeks. The outcome measurements were assessed on weekly basis using PSQI, DASS-21, clinical and biochemical parameters. ANOVA test were used to assess within group differences and ANCOVA were used to check for differences between groups. Significantly lower (better) sleep latency score, sleep disturbances score and daytime dysfunction score were demonstrated on week-4 in intervention group compared to placebo group. Profound differences were observed between the two groups for anxiety and stress scores from week-3 onwards, in which lower anxiety and stress scores were observed in intervention group compared to placebo group. In addition, significantly lower depression score in intervention group compared to placebo group on week-4 was observed. Intervention supplement has demonstrated potent effects in relieving anxiety, stress, depression and sleep quality.
\end{abstract}

\section{INTRODUCTION}

Problems of falling asleep, remaining asleep or waking up too early every morning, are the key symptoms of insomnia. Several studies have demonstrated that approximately $25 \%$ to $36 \%$ of adults in the general population experience occasional or transient insomnia ${ }^{1-6}$. On the other hand, chronic insomnia was indicated in $7.5 \%$ to $15 \%$ of adults. Insomnia may bring to numerous adverse outcomes both at individual and societal levels.

Explicitly, insomnia may lead to daytime dysfunction and fatigue, impaired cognitive functions such as unable to concentrate and loss of memory ${ }^{7-8}$, as well as depression, anxiety and stress 9-11. Specifically in Malaysia, it has been reported that the prevalence of insomnia symptom in the general population was $33.8 \%$, and $12.2 \%$ of them had chronic insomnia ${ }^{14}$. Furthermore, a communitywide study involved 8 countries across African and Asia revealed that $16.6 \%$ out of 43,935 subjects demonstrated severe/extreme nocturnal sleep problems, with a remarkable distinction across the 8 populations, varying from $3.9 \%$ (Purworejo, Indonesia and Nairobi, Kenya) to more than 40\% (Matlab, Bangladesh) ${ }^{15}$.Furthermore, sleep debt may accelerate the development of metabolic disorders such as overweight or obesity, diabetes and high blood pressure ${ }^{12-13}$.
Chronic insomnia are associated with mild depression, anxiety and stress ${ }^{16-17}$. Anxiety and stress may influence some aspects of sleep ${ }^{18}$ such as alterations of sleep/ wake rhythm and changes in sleep duration ${ }^{19}$. Knowing that the chronic insomnia are associated with mild depression, anxiety and stress $^{9,10,20}$, we may stipulate that interventions which alleviate these emotional factors (depression, anxiety and stress) may be advantages to individuals experiencing insomnia; and vice versa.

The benzodiazepines were recommended for the treatment of insomnia and anxiety in the 1960s. They were demonstrated to possess undesirable effects such as ataxia, amnesia, nausea, fatigue, leucopenia, hypotension, confusion, hostility and depression ${ }^{21}$, dependence and abuse ${ }^{22-23}$. However, they are still in use nowadays for the treatment of insomnia albeit less frequent prescribed compared to the past, due to those adverse effects ${ }^{24}$.

Another plausible approach is to concentrate on natural products. Bovine's milk has long been believed by folk wisdom as a soothing or calming beverage aiding sleep. It has been demonstrated that adults taking a meal of milk with cornflakes demonstrated stronger tendency toward uninterrupted sleep ${ }^{25}$, and it was demonstrated that 
evening consumption as well of lactalbumin may enhance sleep quality and daytime alertness ${ }^{26}$.

Hence, for this purpose, a population of Malaysian adults complaining about insomnia or moderate level of depression/ anxiety/ stress were provided intervention over an extended period of four consecutive weeks to investigate the effectiveness of alpha-s1-casein tryptic hydrolysate and Ltheanine on sleep disorder and psychological components. The evolution of sleep parameters was assessed via the Pittsburgh Sleep Quality Index (PSQI). On the other hand, we aimed to compare depression, anxiety and stress scores at baseline through week-4 post-supplementation in addition to blood pressure. Furthermore, hemoglobin level was evaluated at pre-intervention and post-intervention too.

In view of the principal role of iron in the monoamine's metabolism in the brain as well as the role of the monoamines in sleep physiology, it is postulated that sleep quality might associate with hemoglobin level ${ }^{27}$.

\section{METHODOLOGY}

\section{Study design}

A randomized, double-blind, placebo-controlled, parallel group study involving 70 adults with sleep disorders aged $\geq 18$ years was conducted among general population at Kampar district, Perak state of Peninsular Malaysia between November 2016 and July 2017.

\section{Eligibility}

Participants were of either gender aged between 18 and 60 years diagnosed with Pittsburgh Sleep Quality Index (PSQI) score $\geq 5$ or with moderate level of depression/ anxiety/ stress. Participants were otherwise-healthy, free of cardiovascular-related diseases, cancer, diabetes, neurological diseases, or other serious degenerative illnesses and not physically or mentally handicapped. Participants lived in Perak, with no predetermined plans to move to other states during the intervention study. Participants were excluded if they were pregnant; with obstructive sleep apnea; on hypnotics or sleep drugs; psychosis patients, taking sleeping pills or psychotropic drugs; failed to confirm consumption of study capsule; or experienced adverse events that required discontinuation of intervention.

\section{Sample size}

The minimum sample size for the study was calculated using the Altman's nomogram ${ }^{33}$ with $80 \%{ }^{39}$ power (Figure 2 ). Hence, the minimum sample size required would be 35 participants per group to accommodate for $50 \%$ dropout rate.

A third party-controller assigned intervention and placebo capsules to two groups with 1:1 ratio using randomization software. Both researchers and participants were blinded to the allocation. A conscientious and stringent selection process was conducted in the recruitment of participants. Potential participants were broadly screened via surveys to identify eligible participants. Only those who met the strict eligibility criteria were invited to participate in the study.

Participants received once-daily capsules of intervention $(150 \mathrm{mg}$ alpha-s1-casein tryptic hydrolysate and $50 \mathrm{mg}$ L-theanine) or placebo (150 mg skimmed milk powder) for four weeks before bedtime. Alpha s1-casein tryptic hydrolysate is a bioactive peptide extracted from the raw, pure and high-quality milk of only the Holstein breed in France. On the other hand, L-theanine is an aminoacid derived from green tea leaves (Camellia sinensis). Participants reported information regarding their sleep quality, sleep duration, sleep latency, sleep disturbances, habitual sleep efficiency, use of sleeping medication and daytime dysfunction via Pittsburgh Sleep Quality Index questionnaire 28 ; and DASS (depression, anxiety, stress scale) scores $^{29}$ was determined too. Both intervention and placebo capsules were manufactured from cellulose in vegetable. Participants were asked to maintain their usual diet and lifestyle throughout the intervention study.

\section{Ethical consideration}

The study was conducted in accordance with Malaysian Guidelines for Good Clinical Practice and the Declaration of Helsinki. The protocol was approved by the University Tunku Abdul Rahman Scientific and Ethical Review Committee (Research Project Identification No. U/SERC/67/2016). All participants gave written informed consent. 


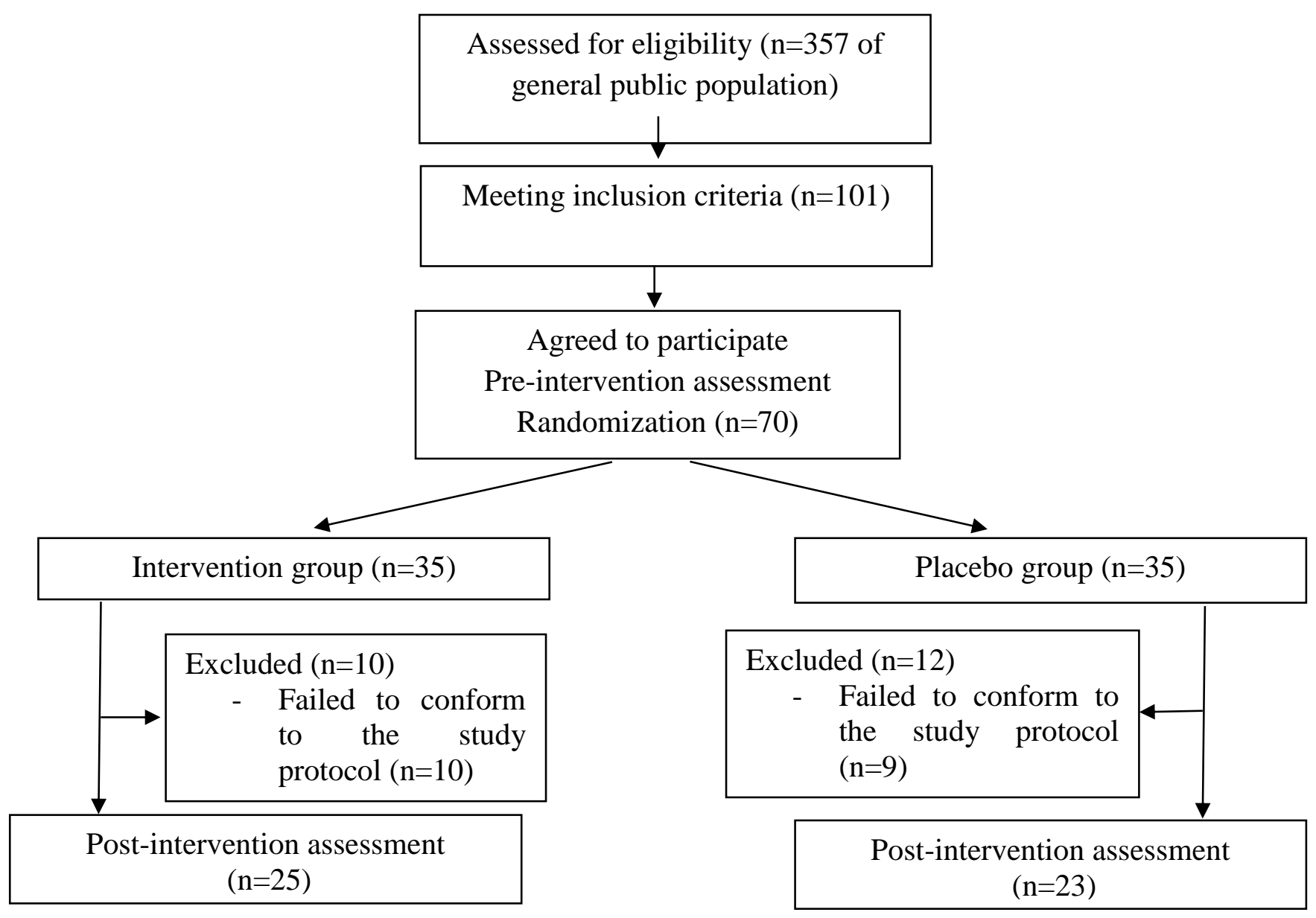

Figure 1: Recruitment and progress of participants through study

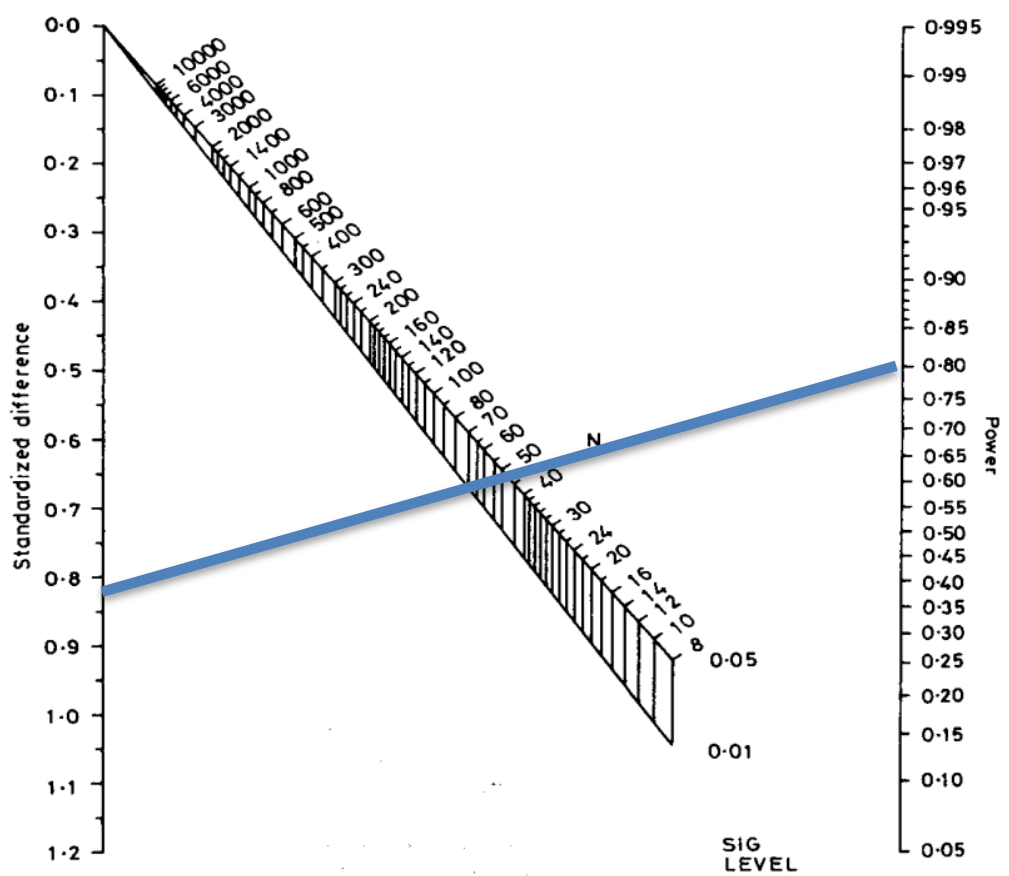

Figure 2: Altman's nomogram 
Safety considerations

Adverse outcomes that happened during the study were recorded. Participants will not be obliged to avoid from administering sleeping pills if medically indicated by healthcare professional.

\section{Study agents}

The test capsules consisted of $150 \mathrm{mg}$ alpha-s1casein tryptic hydrolysate and $50 \mathrm{mg}$ L-theanine (intervention group) and $150 \mathrm{mg}$ skimmed milk powder (placebo group) prepared by the manufacturer (LiveLife Sdn. Bhd.). Both the test capsules and placebo capsules were encapsulated from cellulose in vegetable and filled in unmarked individual bottles. The formulation and manufacturing of the test capsules has been approved as health supplement by Ministry of Health Malaysia.

\section{Efficacy measurements}

The Pittsburgh Sleep Quality Index (PSQI) ${ }^{28}$ is a 21item self-administered instrument, which yields a global score. It represents the sum of seven individual component scores: subjective sleep quality, sleep latency, sleep duration, habitual sleep efficacy, sleep disturbances (including items such as feeling pain, having difficulty breathing), use of medications for sleep and daytime dysfunction (including items related to daytime sleepiness and energy).

DASS scores ${ }^{29}$ was also assessed using DASS-21 selfadministered questionnaire, which is the shorter form of the original DASS-42 questionnaire which measures negative emotional states of depression, anxiety and stress ${ }^{31}$. This instrument contains three sections of 7-item self-report scales for measuring anxiety, depression and stress. Each subscale was scored on a 4-point scale from 0 (not present) to 3 (considerable). This scale measures the extent to which each state has been experienced "over the past 2 days". Scores for the scales were determined by summing the raw scores for the relevant 7 items; the scores for each subscale were then multiplied by two because DASS-21 is truncated version of DASS-42, this multiplication procedure was performed to ensure comparability of outcomes to DASS-42 in accordance to the manual ${ }^{32}$. The sum of scores for each subscale after multiplication ranged from 0 (no symptom) to 42 (high level of symptoms).

Duplicate measurements of blood pressure (BP in $\mathrm{mmHg}$ ) was measured two minutes apart with a digital automated blood pressure monitor, after the participants have rested in a seated position for five minutes. An average of the two readings was taken weekly ${ }^{30}$.

Finger pricking was conducted on the participants. Their hemoglobin level was measured by using Mission $®$ Plus Hemoglobin Meter (Acon Laboratory,
United States). A drop of blood $(10 \mu \mathrm{L})$ was added to the test strip, and the result was displayed on the screen in 15 seconds.

\section{Statistical analysis}

An independent samples $t$-test was used to determine if any significant differences in the mean of the continuous variables existed between those participants in intervention and placebo group. In the case where significant difference was found at baseline, an ANCOVA was used with baseline data as covariate (latency, habitual sleep efficiency, diastolic blood pressure). A general linear model (GLM) repeated-measures ANOVA for the two groups over baseline, week-1, week-2, week-3 and week-4 was used to determine if any significant differences existed within groups over time for selected variables. Data were analyzed using SPSS version 22. A statistical probability level of $\mathrm{p}<0.05$ (two-sided) was considered as significant. The data were performed on an intention-to-treat (complete-case analysis) basis.

\section{RESULT}

Of the 70 eligible participants who underwent randomization, 35 were assigned to each intervention and placebo groups, with 10 and 12 loss to follow-up at the end of the study in intervention and placebo groups, respectively. There were no significant adverse effects reported until the end of the study. The baseline demographic characteristics were compatible (Table 1).

Table 1: Socio-demographic data of participants $(\mathrm{n}=48)$

\begin{tabular}{lccc}
\hline Parameter & $\begin{array}{c}\text { Intervention } \\
\mathbf{2 5}\end{array}$ & $\begin{array}{c}\text { Control } \\
\mathbf{2 3}\end{array}$ & $\begin{array}{c}\text { p- } \\
\text { value }\end{array}$ \\
\hline Gender & & & 0.868 \\
Male & $6(24.0)$ & $6(26.1)$ & \\
Female & $19(76.0)$ & $17(73.9)$ & \\
Age (Years) & $30.40 \pm 7.51$ & $31.17 \pm 7.33$ & 0.720 \\
Ethnicity & & & 0.668 \\
Malays & $0(0.0)$ & $0(0.0)$ & \\
Chinese & $21(84.0)$ & $21(91.3)$ & \\
Indian & $4(16.0)$ & $2(8.7)$ & \\
\hline
\end{tabular}

The results of the statistical comparisons in participants for the Pittsburg Sleep Quality Index (PSQI) score, between intervention and placebo groups, on week-0 (baseline) to week-4 postsupplementation were demonstrated (Table 2). Profound differences were observed between the two groups for habitual sleep efficiency from week1 onwards. On the other hand, there was also significant differences found between the two groups for sleep duration, sleep latency and sleep disturbances at week-1, week-2 and week-3, respectively. 
Table 2: Pittsburg Sleep Quality Index (PSQI) scores

\begin{tabular}{|c|c|c|c|}
\hline Parameter & $\begin{array}{c}\text { Intervention } \\
\text { Mean } \pm \text { SD }\end{array}$ & $\begin{array}{c}\text { Control } \\
\text { Mean } \pm \text { SD }\end{array}$ & $\begin{array}{c}\text { p-value } \\
\text { (Between-group) }\end{array}$ \\
\hline Total number of participants & 25 & 23 & \\
\hline \multicolumn{4}{|l|}{ PSQI global score } \\
\hline aPre-intervention (Week-0) & $7.24 \pm 2.71$ & $6.13 \pm 2.18$ & 0.127 \\
\hline aPost-intervention (Week-1) & $5.52 \pm 2.62$ & $6.70 \pm 3.08$ & 0.160 \\
\hline aPost-intervention (Week-2) & $5.52 \pm 2.50$ & $5.70 \pm 3.36$ & 0.837 \\
\hline aPost-intervention (Week-3) & $4.52 \pm 2.18$ & $5.96 \pm 3.36$ & 0.083 \\
\hline aPost-intervention (Week-4) & $4.52 \pm 2.65$ & $5.78 \pm 1.93$ & 0.674 \\
\hline P-value (Within-group) & $0.003^{*}$ & 0.342 & \\
\hline \multicolumn{4}{|l|}{ Sleep quality } \\
\hline aPre-intervention (Week-0) & $1.28 \pm 0.94$ & $1.35 \pm 0.89$ & 0.798 \\
\hline aPost-intervention (Week-1) & $0.80 \pm 087$ & $1.13 \pm 0.92$ & 0.206 \\
\hline aPost-intervention (Week-2) & $0.88 \pm 0.83$ & $0.74 \pm 0.86$ & 0.568 \\
\hline aPost-intervention (Week-3) & $0.88 \pm 0.93$ & $0.87 \pm 1.06$ & 0.971 \\
\hline aPost-intervention (Week-4) & $0.92 \pm 0.64$ & $0.83 \pm 0.58$ & 0.597 \\
\hline P-value (Within-group) & 0.114 & $0.001^{*}$ & \\
\hline \multicolumn{4}{|l|}{ Latency } \\
\hline aPre-intervention (Week-0) & $1.96 \pm 0.89$ & $1.35 \pm 0.832$ & $0.018^{*}$ \\
\hline bPost-intervention (Week-1) & $1.04 \pm 0.74$ & $1.17 \pm 0.65$ & 0.803 \\
\hline bPost-intervention (Week-2) & $1.00 \pm 0.58$ & $0.70 \pm 0.56$ & $0.029^{*}$ \\
\hline bost-intervention (Week-3) & $0.72 \pm 0.68$ & $0.91 \pm 0.60$ & 0.482 \\
\hline 'Dost-intervention (Week-4) & $0.72 \pm 0.46$ & $0.91 \pm 0.29$ & $0.029^{*}$ \\
\hline P-value (Within-group) & $<0.001^{*}$ & $0.021^{*}$ & \\
\hline \multicolumn{4}{|l|}{ Duration } \\
\hline aPre-intervention (Week-0) & $1.48 \pm 0.87$ & $1.52 \pm 0.51$ & 0.839 \\
\hline aPost-intervention (Week-1) & $1.04 \pm 1.02$ & $1.74 \pm 0.69$ & $0.008^{*}$ \\
\hline aPost-intervention (Week-2) & $1.12 \pm 1.17$ & $1.13 \pm 0.82$ & 0.971 \\
\hline aPost-intervention (Week-3) & $1.28 \pm 1.10$ & $1.35 \pm 1.03$ & 0.827 \\
\hline aPost-intervention (Week-4) & $1.24 \pm 1.09$ & $1.13 \pm 0.34$ & 0.637 \\
\hline P-value (Within-group) & $0.044^{*}$ & $<0.001^{*}$ & \\
\hline \multicolumn{4}{|l|}{ Habitual sleep efficiency } \\
\hline aPre-intervention (Week-0) & $0.24 \pm 0.44$ & $0.00 \pm 0.00$ & $0.011^{*}$ \\
\hline bPost-intervention (Week-1) & $0.36 \pm 0.49$ & $0.39 \pm 0.72$ & $0.009^{*}$ \\
\hline bPost-intervention (Week-2) & $0.60 \pm 0.71$ & $0.13 \pm 0.34$ & $0.003^{*}$ \\
\hline 'Post-intervention (Week-3) & $0.36 \pm 0.49$ & $0.26 \pm 0.45$ & $<0.001^{*}$ \\
\hline bPost-intervention (Week-4) & $0.28 \pm 0.46$ & $0.22 \pm 0.42$ & $0.333^{*}$ \\
\hline P-value (Within-group) & $0.024^{*}$ & $0.011^{*}$ & \\
\hline \multicolumn{4}{|l|}{ Use of medications } \\
\hline aPre-intervention (Week-0) & $0.00 \pm 0.00$ & $0.13 \pm 0.46$ & 0.186 \\
\hline aPost-intervention (Week-1) & $0.36 \pm 1.00$ & $0.26 \pm 0.62$ & 0.684 \\
\hline aPost-intervention (Week-2) & $0.48 \pm 1.05$ & $0.52 \pm 0.90$ & 0.883 \\
\hline aPost-intervention (Week-3) & $0.36 \pm 1.00$ & $0.39 \pm 0.72$ & 0.902 \\
\hline aPost-intervention (Week-4) & $0.36 \pm 1.00$ & $0.26 \pm 0.45$ & 0.663 \\
\hline P-value (Within-group) & $0.038^{*}$ & 0.270 & \\
\hline \multicolumn{4}{|l|}{ Disturbances } \\
\hline aPre-intervention (Week-0) & $1.08 \pm 0.28$ & $0.83 \pm 0.65$ & 0.094 \\
\hline aPost-intervention (Week-1) & $0.92 \pm 0.49$ & $1.00 \pm 0.62$ & 0.624 \\
\hline aPost-intervention (Week-2) & $0.88 \pm 0.53$ & $0.91 \pm 0.67$ & 0.849 \\
\hline aPost-intervention (Week-3) & $0.64 \pm 0.57$ & $1.13 \pm 0.87$ & $0.024^{*}$ \\
\hline aPost-intervention (Week-4) & $0.64 \pm 0.49$ & $1.39 \pm 0.50$ & $<0.001^{*}$ \\
\hline P-value (Within-group) & $0.009^{*}$ & $0.029^{*}$ & \\
\hline \multicolumn{4}{|l|}{ Daytime dysfunction } \\
\hline aPre-intervention (Week-0) & $1.20 \pm 1.23$ & $0.96 \pm 0.71$ & 0.399 \\
\hline aPost-intervention (Week-1) & $1.00 \pm 0.96$ & $1.04 \pm 0.83$ & 0.867 \\
\hline aPost-intervention (Week-2) & $0.56 \pm 0.71$ & $1.57 \pm 1.16$ & $0.001^{*}$ \\
\hline aPost-intervention (Week-3) & $0.28 \pm 0.46$ & $1.04 \pm 0.93$ & $0.001^{*}$ \\
\hline aPost-intervention (Week-4) & $0.36 \pm 1.00$ & $1.04 \pm 0.88$ & $0.002^{*}$ \\
\hline P-value (Within-group) & $<0.001^{*}$ & $0.049^{*}$ & \\
\hline
\end{tabular}


Daytime dysfunction score was significantly different between the two groups at week-2 onwards. Specifically, significantly lower daytime dysfunction and sleep disturbances scores were observed in intervention group compared to placebo group on week-2 and week-3 onwards, respectively. Significantly lower (better) sleep latency score, sleep disturbances score and daytime dysfunction score were demonstrated on week-4 in intervention group compared to placebo group.

In addition, depression, anxiety and stress scores, between intervention and placebo groups, on week0 (baseline) to week-4 post-supplementation were demonstrated (Table 3 ). Profound differences were observed between the two groups for anxiety and stress scores from week-3 onwards, in which lower anxiety and stress scores were observed in intervention group compared to placebo group. In addition, significantly lower depression score in intervention group compared to placebo group on week- 4 was observed. On the other hand, there was also significant difference found between the two groups for diastolic blood pressure from week-1 onwards. PSQI global score, use of medications score, anxiety score, stress scores were significantly improved over time in intervention group; whilst no significant change was observed in placebo group over time.

Table 3: Depression, anxiety and stress scores

\begin{tabular}{lccc}
\hline Parameter & $\begin{array}{c}\text { Intervention } \\
\text { Mean } \pm \text { SD }\end{array}$ & $\begin{array}{c}\text { Control } \\
\text { Mean } \pm \text { SD }\end{array}$ & P-value \\
\hline Total number of participants & 25 & 23 & \\
Depression & $7.92 \pm 9.23$ & $9.65 \pm 8.46$ & 0.502 \\
aPre-intervention (Week-0) & $6.40 \pm 10.61$ & $7.91 \pm 7.25$ & 0.570 \\
aPost-intervention (Week-1) & $4.56 \pm 7.41$ & $8.87 \pm 9.04$ & 0.076 \\
aPost-intervention (Week-2) & $2.80 \pm 7.72$ & $8.17 \pm 10.46$ & 0.051 \\
aPost-intervention (Week-3) & $2.96 \pm 8.29$ & $8.78 \pm 9.72$ & $0.030^{*}$ \\
aPost-intervention (Week-4) & $<0.001^{*}$ & $0.037^{*}$ & \\
P-value (Within-group) & & & \\
Anxiety & $9.36 \pm 5.68$ & $9.48 \pm 5.33$ & 0.941 \\
aPre-intervention (Week-0) & $6.80 \pm 7.33$ & $10.09 \pm 9.75$ & 0.191 \\
aPost-intervention (Week-1) & $4.40 \pm 5.03$ & $8.52 \pm 9.58$ & 0.074 \\
aPost-intervention (Week-2) & $2.40 \pm 4.28$ & $6.78 \pm 8.63$ & $0.035^{*}$ \\
aPost-intervention (Week-3) & $1.92 \pm 3.34$ & $7.30 \pm 8.33$ & $0.007^{*}$ \\
aPost-intervention (Week-4) & $<0.001^{*}$ & 0.146 & \\
P-value (Within-group) & & & \\
Stress & $15.52 \pm 7.73$ & $15.13 \pm 9.28$ & 0.875 \\
aPre-intervention (Week-0) & $11.60 \pm 10.74$ & $12.43 \pm 10.37$ & 0.786 \\
aPost-intervention (Week-1) & $8.00 \pm 8.29$ & $12.70 \pm 10.54$ & 0.092 \\
aPost-intervention (Week-2) & $4.88 \pm 6.27$ & $10.61 \pm 11.85$ & $0.047^{*}$ \\
aPost-intervention (Week-3) & $4.48 \pm 6.36$ & $9.74 \pm 10.52$ & $0.045^{*}$ \\
aPost-intervention (Week-4) & $<0.001^{*}$ & 0.065 & \\
P-value (Within-group) & &
\end{tabular}

\section{DISCUSSION}

In this randomized preliminary study, the two groups were well balanced for baselinedemographics. After four-week intervention, intervention group demonstrated remarkable effects, at least in the short-term, in alleviating some sleep disorder symptoms relative to the placebo, specifically daytime dysfunction and sleep disturbances. The placebo group also showed a lower sleep latency and habitual sleep efficiency scores as well as diastolic blood pressure at week2 , which may be due to the placebo effect.

The observed properties of intervention capsules may be due to its main chemical ingredients, which include alpha-s1-casein tryptic hydrolysate and L- theanine. Although our study could not determine which specific ingredient (s) was responsible for the observed effect, there are several possible modes of action that might have accountable for producing our recorded results. For instance, L-theanine, extracted from green tea, may affect the secretion of neurotransmitters in the brain ${ }^{34}$, antagonize caffeine-caused excitation and relieve psychological tension ${ }^{35}$. Alpha-s1-casein tryptic hydrolysate has been reported to exhibit a benzodiazepine-like activity of the $\mathrm{GABA}_{A}$ receptor without side effects ${ }^{36}$. At present, the exact mechanism of action of alpha-s1-casein tryptic hydrolysate on sleep disorders is not known. Given the anti-stress properties of alpha-s1-casein tryptic hydrolysate, it seems possible to relate the detected improvement of sleep aspects to a 
reduction of stress following the chronic administration of the treatment. Further studies will be necessary in order to clarify this essential aspect of alpha-s1-casein tryptic hydrolysate properties on sleep.

It is interesting to point out that the score of some of the assessed depression, anxiety and stress scores was decreased also in participants treated by placebo. Depression, anxiety and stress are well known for having an important psychological component and placebo response is often very high in clinical studies concerning them ${ }^{37}$. The "HURIETSERUSCLAT" law, which is concerned with trials on human subjects in France, implies that the participants sign an "informed consent" form before the beginning of the research. This means that when the study begins, they know that they will be treated by a substance (intervention or placebo), and that intervention capsules can possibly reverse their symptoms of depression, anxiety and stress. Participation in this study might have conferred a placebo effect.

The placebo (skimmed milk powder) contains casein and particularly alpha-s1-casein (entire proteins), but the bioactive peptide identified in the alpha-s1casein tryptic hydrolysate, namely alpha-s1-casein (f91-100) cannot be significantly released (from it) because in vivo digestion can be assimilated as a multi-enzymatic process including first a pepsic hydrolysis in the stomach. Pepsin cleaves 91-92, 9596, $98-99$ and $99-100$ bonds of alpha-s1-casein ${ }^{38}$ preventing alpha-s1-casein (f91-100) release from the entire protein contained in placebo. The significant content of the bioactive peptide in the alpha-s1-casein tryptic hydrolysate and its relative small size may protect it from further digestion and may explain the significant effects after in vivo intake. In this study, the alpha-s1-casein tryptic hydrolysate results from a mono-enzymatic digestion of purified alpha-s1-casein.

Table 4: Biochemical and clinical parameters

\begin{tabular}{lccc}
\hline Parameter & $\begin{array}{c}\text { Intervention } \\
\text { Mean } \pm \text { SD }\end{array}$ & $\begin{array}{c}\text { Control } \\
\text { Mean } \pm \text { SD }\end{array}$ & P-value \\
\hline Total number of participants & 25 & 23 & \\
Systolic Blood pressure (mmHg) & & & \\
aPre-intervention (Week-0) & $109.48 \pm 11.72$ & $112.85 \pm 12.24$ & 0.335 \\
aPost-intervention (Week-1) & $111.36 \pm 10.07$ & $113.09 \pm 9.77$ & 0.550 \\
aPost-intervention (Week-2) & $110.10 \pm 7.73$ & $108.98 \pm 8.49$ & 0.634 \\
aPost-intervention (Week-3) & $106.32 \pm 7.59$ & $105.52 \pm 11.40$ & 0.779 \\
aPost-intervention (Week-4) & $115.06 \pm 11.46$ & $109.72 \pm 15.22$ & 0.174 \\
P-value (Within-group) & $0.001^{*}$ & $<0.001^{*}$ & \\
Diastolic Blood pressure (mmHg) & & & \\
aPre-intervention (Week-0) & $64.80 \pm 6.96$ & $69.50 \pm 6.75$ & $0.022^{*}$ \\
bPost-intervention (Week-1) & $64.54 \pm 5.44$ & $66.94 \pm 4.53$ & $0.045^{*}$ \\
bPost-intervention (Week-2) & $65.54 \pm 7.64$ & $64.54 \pm 5.80$ & $0.001^{*}$ \\
bPost-intervention (Week-3) & $62.84 \pm 5.70$ & $64.39 \pm 8.51$ & $0.002^{*}$ \\
bPost-intervention (Week-4) & $68.40 \pm 6.32$ & $67.70 \pm 8.51$ & $0.005^{*}$ \\
P-value (Within-group) & $<0.001^{*}$ & $<0.001^{*}$ & \\
Hemoglobin & & & \\
aPre-intervention (Week-0) & $13.28 \pm 1.42$ & $13.72 \pm 2.23$ & 0.422 \\
aPost-intervention (Week-4) & $12.98 \pm 1.26$ & $13.15 \pm 1.24$ & 0.653 \\
P-value (Within-group) & 0.427 & 0.146 & \\
\hline
\end{tabular}

${ }^{a}$ Analysis based on independent samples t-test

${ }^{b}$ Analysis based on Analysis of Covariance (Baseline data was used as covariate to adjust for the baseline difference for sleep latency, habitual sleep efficiency, diastolic blood pressure)

PSQI dimensional scores can range from 0 to 3 . Higher score indicates poorer sleep quality in the dimension

\section{CONCLUSION}

In conclusion, this study provided evidence that a four-week ingestion of alpha-s1-casein tryptic hydrolysate and L-theanine displayed positive effect in individuals with self-reported complaints suggesting that alpha-s1-casein tryptic hydrolysate and L-theanine may be used as an effective functional ingredient alleviating sleep disorders specifically daytime dysfunction and sleep disturbances as well as depression, anxiety and stress. Further studies are needed to investigate if the conclusions drawn from this population can be generalized to other ethnicities, and if the effects can be observed on a long-term basis.

\section{ACKNOWLEDGEMENT}

This study was funded by LiveLife Biosciences AG (Research project vote account number 4483/000). Intervention and placebo pills were provided by LiveLife Biosciences AG, Switzerland. 


\section{REFERENCES}

1. Dodge, R; Cline, MG; Quan, SF. The natural history of insomnia and its relationship to respiratory symptoms. Arch Intern Med 1995; 155: 1797-1800.

2. Ancoli-Israel, S; Roth, T. Characteristics of insomnia in the United States: results of the 1991 National Sleep Foundation Survey. Sleep 1999; 22(Suppl 2): S347S353.

3. Bixler, EO; Vgontzas, AN; Lin HM; VelaBueno, A; Kales, A. Insomnia in central Pennsylvania. J Psychosom Res 2002; 53: 589-592.

4. Ohayon, MM; Guilleminault, C; Paiva, T; Priest, RG; Rapoport, DM; Sagales, T; Smirne, S; Zulley, J. An international study on sleep disorders in the general population: methodological aspects of the use of the Sleep-EVAL system. Sleep 1997; 20: 1086-1092.

5. Ohayon, MM; Caulet, M; Priest, RG; Guilleminault, C. DSM-IV and ICSD-90 insomnia symptoms and sleep dissatisfaction. Br J Psychiatry 1997; 171: 382-388.

6. Ohayon, MM; Caulet, M; Guilleminault, C. How a general population perceives its sleep and how this relates to the complaint of insomnia. Sleep 1997; 20: 715-723.

7. Roth, T; Ancoli-Israel, S. Daytime consequences and correlates of insomnia in the United States: results of the 1991 National Sleep Foundation Survey. II. Sleep 1999; 22 (Suppl 2): S354-S358.

8. Thase, ME. Correlates and consequences of chronic insomnia. Gen Hospital Psychiatry 2005; 27: 100-12.

9. Perlis, ML; Giles, DE; Mendelson, WB; Bootzin, RR; Wyatt, JK. Psychophysiological insomnia: the behavioral model and a neurocognitive perspective. J Sleep Res 1997; 6: 179-188.

10. Sukegawa, T; Itoga, M; Seno, H; Miura, S; Inagaki, T; Salto, W; et al. Sleep disturbances and depression in the elderly in Japan. Psychiatry Clin Neurosci 2003; 57: 265-70.
11. Kaneita, Y; Uchiyama, M; Takemura, S; Yokoyama, E; Miyake, T; Harano, S; et al. Use of alcohol and hypnotic medication as aids to sleep among the Japanese general population. Sleep Med 2007; 8: 723-732.

12. Spiegel, K; Leproult, R; Van Cauter, E. Impact of sleep debt on metabolic and endocrine function. Lancet 1999; 4: 4359.

13. Hayashino, Y; Fukuhara, S; Suzukamo, Y; Okamura, T; Tanaka, T; Ueshima, H. Relation between sleep quality and quantity, quality of life, and risk of developing diabetes in healthy workers in Japan: the High-risk and Population Strategy for Occupational Health Promotion (HIPOP-OHP) Study. BMC Public Health 2007; 7: 129.

14. Zailinawati, AH; Ariff KM; Nurjahan Ml; Teng CL. Epidemiology of insomnia in Malaysian adults: A community-based survey in 4 urban areas. Asia Pacific Journal of Public health, 2008; 20(3): 2240-233.

15. Saverie, S; Wiliam, T; Francesc, XG; Margaret, T; Ngianga-Bakwin K. Sleep problems: An emerging global epidemic? Findings from the INDEPTH WHO-SAGE study among more than 40,000 older adults from 8 countries across Africa and Asia. Sleep 2012; 35(8): 1173-1181.

16. Benca, RM; Mood disorders. In: Kryger, T; Dement, WC; editors. Principles and practice of sleep medicine. 2000; Philadelphia: WB Saunders, pp. 1140-57.

17. De Saint Hilaire, Z; Straub, J; Pelissolo, A. Temperament and character in primary insomnia. Eur Psychiatry 2005; 20: 188-92.

18. Vollrath, M; Wicki, W; Angst, J. The Zurich study. VIII. Insomnia: association with depression, anxiety, somatic syndromes, and course of insomnia. Eur Arch Psychiatry Neurol Sci 1989; 239: 113-24.

19. Bonnet, MH; Arand, DL. Hyperarousal and insomnia. Sleep Med Rev 1997; 1: 97-108.

20. Hall, M; Buysse, DJ; Nowell, PD; Nofzinger, EA; Houck, P; Reynolds, CF; Kupfer, DJ. Symptoms of stress and depression as correlates of sleep in primary insomnia. Psychosom Med 2000; 62: 227-30. 
21. Olajide, D; Lader, M. Depression following withdrawal from long term benzodiazepine use: a report of four cases. Psychol Med 1984; 14: 937-040.

22. Power, KG; Jerrom, DW; Simpson, RJ; Mitchell, M. Controlled study of withdrawal symptoms and rebound anxiety after six week course of diazepam for generalised anxiety. Br Med J (Clin Res Ed) 1985; 290: 1246-1248.

23. Rosenberg, HC; Chiu, TH. Time course for development of benzodiazepine tolerance and physical dependence. Neurosci Biobehav Rev 1985; 9: 123-31.

24. Staner, L; Ertlé, S; Boeijinga P; Rinaudo, G; Arnal, MA; Muzet, A; Lutringer, R. Nextday residual effects of hypnotics in DSM-IV primary insomnia: a driving simulator study with simultaneous electroencephalogram monitoring. Psychopharmacology (Berl) 2005; 181: 790-8.

25. Laird, DA; Drexel, H. Experimenting with food and sleep. I. Effects of varying types of foods in offsetting sleep disturbances caused by hunger pangs and gastric distress-children and adults. J Am Diet Assoc 1934; 10: 89-94.

26. Markus, CR; Jonkman, LM; Lammers, JH; Deutz, NE; Messer, MH; Rigtering, NE. Evening Intake of Lactalbumin May Improve Sleep and Morning Alertness. Am J Clin Nutr 2005; 81: 1026-33.27.

27. Semiz, M; Uslu, A; Korkmaz, S; Demir, S; Parlak, I; Sencan, M; Aydin, B; Uncu, T. Assessment of subjective sleep quality in iron deficiency anaemia. Afr. Health Sci 2015; 15(2): 621-627.

28. The Pittsburgh Sleep Quality Index (PSQI). Available from: http:/ / www.sleep.pitt.edu/content.asp?i $d=1484$ \&subid=2316.

29. Lovibond, PF; Lovibond, S. Manual for the Depression Anxiety Stress Scales. 1995; Sydney, Australia: School of Psychology, University of New South Wales.

30. Murphy, MH; Murtagh, EM; Boreham, CAG; Hare, LG; Nevill, AM. The effect of a worksite based walking programme on cardiovascular risk in previously sedentary civil servants. BMC Public Health 2006; 6(1): 136-143.
31. Musa, R; Fadzil, MA; Zain, Z. Translation, validation and psychometric properties of Bahasa Malaysia version of the Depression, Anxiety and Stress (DASS). ASEAN Journal of Psychiatry 2007; 8(2): 82-89.

32. Ariaratnam, S; Devi, A; Kaur, G; Sinniah, D; Suleiman, A; Thambu, $M$; et al. Psychiatric morbidity and survival in newly diagnosed treatment naïve cancer patients- A study from Malaysia. Biomed Res. 2008; 19(2): 113-116.

33. Altman, Douglas G. Practical statistic for medical research. Monographs on Statistics and Applied Probability (First Edition). 1991; Chapman and Hall.

34. Yamaada, T; Terashima, T; Okubo, T; Junela, LR; Yokogoshi, H. Effects of theanine, -glutamyethylamide, on neurotransmitter release and its relationship with glutamic acid neurotransmission. Nutr. Neurosci. 2005; 8: 219-226.

35. Kamath, AB; Wang, L., Das, H; Li, L; Reinhold, VN, Bukowski, JF. Antigens in tea-beverage prime human Vgamma $2 v$ delta $2 \mathrm{~T}$ cells in vitro and in vivo for memory and nonmemory antibacterial cytokine responses. Proc. Nat. Acad. Sci. USA 2003; 100: 6009.

36. Miclo, L; Perrin, E; Driou, A; Papadopoulous, V; Boujrad, N; Vanderesse, R; Boudler, JF; Desor D; Linden, G; Gallard, JL. Characterization of alpha-casozepine, a tryptic peptide from bovine alpha(s1)-casein with benzodiazepine-like activity. FASEB J 2001; 15(10): 1780-1782.

37. Berk, M; Dodd, S. Antidepressants and the placebo response. Hum Psychopharmacol 2005; 5: 305-307.

38. Mercier, JC; Grosclaude, F; RibadeauDumas B. Primary structure of bovin alpha-s1-casein partial sequence. Eur $J$ Biochem 1970; 16(3): 453-460.

39. Carmen, R.W., Van, V. Betsy L.M. (2007). Understanding power and rules of thumb for determining sample sizes. Tutorials in Quantitative Methods for Psychology; 3(2): 43-50. 